\title{
Perspectivas existentes frente a la didáctica específica de la filosofía
}

\author{
A Study on Existing \\ Perspectives about \\ Philosophical \\ Didactics
}

\author{
Perspectivas \\ existentes sobre a \\ didática específica \\ da filosofia
}

John Alexander Giraldo Botero* https://orcid.org/0000-0003-0962-1869

Liliana del Pilar Gallego Castaño** https://orcid.org/0000-0001-5300-0213

Francisco Javier Ruiz Ortega*** https://orcid.org/0000-0003-1592-5535

\section{Para citar este artículo}

Giraldo Botero, J., Gallego Cataño, L. y Ruiz Ortega, F. (2021). Perspectivas existentes frente a la didáctica específica de la filosofía. Folios,

(54). https://doi.org/10.17227/folios.54-12162

* Magíster en Educación. Docente IE Escuela Normal Superior María Escolástica, Manizales, Colombia.

Correo: al.exon221@gmail.com

** Doctora en Psicología de la Educación. Docente Departamento de Lenguas Extranjeras, Universidad de Caldas, Manizales, Colombia.

Correo: liliana.gallego@ucaldas.edu.co

*** Doctor en Didáctica de las Ciencias Exactas y las Matemáticas. Docente Departamento Estudios Educativos, Universidad de Caldas, Manizales, Colombia.

Correo: francisco.ruiz@ucaldas.edu.co

Artículo recibido $26 \cdot 07 \cdot 2020$

Artículo aprobado $28 \cdot 01 \cdot 2021$ 


\title{
Resumen
}

Este artículo de investigación tiene como objetivo identificar las concepciones sobre la didáctica de la filosofía considerada como una didáctica disciplinar con un objeto propio de estudio, en los documentos escritos de los programas de las universidades acreditadas en alta calidad para el año 2018 en Colombia, que orientan esta misma asignatura en sus currículos. Para este propósito se analizó el discurso escrito de cinco documentos provistos por las mismas universidades participantes, a través de un enfoque cualitativo y bajo un tipo de estudio basado en el análisis documental. Solo se tuvieron en cuenta dos secciones de la totalidad de la estructura de los programas: objetivos y justificación. Los resultados indican que hay una tendencia a ver en la didáctica de la filosofía un elemento metodológico; es decir, una perspectiva clásica que se tiene sobre la didáctica. Se desconocen, así, elementos como el aprendizaje y el pensamiento crítico que han hecho de este concepto un campo de estudio más amplio. De esta forma, se concluye que debe haber una mayor preocupación desde la didáctica específica de la filosofía por integrar este tipo de procesos a su dinámica epistemológica, de manera que los procesos educativos en este ámbito no se sitúen exclusivamente en el qué enseñar; sino, a su vez, en el cómo se aprende.

\section{Palabras clave}

didáctica; didáctica de la filosofía; estrategias de enseñanza; aprendizaje; pensamiento crítico

\begin{abstract}
This research article attempts to identify the existing conceptions about the Didactics of Philosophy considered like a Disciplinary Didactics with an object of study in the syllabi of high-quality and accredited universities in the year 2018, in Colombia. To do that, a qualitative approach with a documentary analysis was undertaken in this article, only considering two sections in said syllabi: objectives and rationale sections. Results suggest there is a trend towards viewing the Didactics of Philosophy as a methodological element closely linked to a traditional view of didactics ignoring other important elements such as learning and critical thinking considered in contemporary perspectives. In the end, this paper leads to conclude that Didactics of Philosophy should have a greater concern for the integration of those processes to its epistemology in order to focus not only on what to teach but also on how to learn.

\section{Keywords \\ didactics; didactics of philosophy; teaching strategies; learning; critical thinking}

Este artigo de pesquisa tem como objetivo identificar os conceitos da didática da filosofia, considerada disciplinar com seu próprio objeto de estudo, nos documentos dos programas das universidades credenciadas em alta qualidade para 0 ano de 2018 na Colômbia que orientam essa disciplina em seus currículos. Para tanto, analisou-se o discurso escrito de cinco documentos fornecidos pelas universidades participantes, através de uma abordagem qualitativa e sob um tipo de estudo baseado na análise documental. Apenas duas seções de toda a estrutura do programa foram consideradas: objetivos e justificativa. Os resultados indicam que há uma tendência a ver na didática da filosofia um elemento metodológico, sendo essa uma perspectiva clássica que é adotada na didática. Isso ignora elementos como o aprendizado e o pensamento crítico que tornaram esse conceito um campo de estudo mais amplo. Dessa forma, conclui-se que é preciso uma maior preocupação da didática específica da filosofia em integrar esse tipo de processo em sua dinâmica epistemológica, de tal maneira que os processos educacionais nesse campo não se localizem exclusivamente no que ensinar; mas, por sua vez,
\end{abstract}

Abstract

\section{Resumo} na maneira como aprendem.

\section{Palavras chave}

didática; didática da filosofia; estratégias de ensino; aprendizagem; pensamento crítico 


\section{Introducción}

En el contexto de la filosofía aún no existen posiciones claras que permitan asumir un consenso que legitime la existencia de una didáctica específica en esta área del conocimiento. Esto se confirma cuando en las producciones académicas que han existido al respecto hay tendencias que difieren entre sí. Hay quienes toman la didáctica de la filosofía como una herramienta metodológica (Bondy,1967; Araya, 2003; Perelló, 2008; MEN, 2010) y para quienes esta existe como un área que toma en cuenta los diferentes elementos que se agrupan dentro del ejercicio de la práctica educativa (Boavida, 2006; Cerletti, 2008; Gómez, 2003; Kohan, 2011; Obiols, 2008).

Asimismo, cuando se aborda el campo asociado a la enseñanza de la filosofía saltan a la vista algunas preguntas que son necesarias para pensar de manera filosófica la praxis educativa en esta área del conocimiento. Interrogantes relacionados con el porqué y para qué enseñarla, buscan reconfigurar constantemente el ejercicio que adopta el docente en función de su tarea formativa. Esta forma de asumir la enseñanza en un área específica como la filosofía la han adoptado teóricos que también sugieren efectuar el cuestionamiento en torno a qué es filosofía, pues de tener una posición más o menos clara con respecto a lo que significa este saber, se podrían direccionar prácticas de enseñanza y aprendizaje que estuvieran más acordes a ese posicionamiento inicial en torno a esta pregunta (Gómez, 2003; Cerletti, 2008).

En el presente artículo entonces, se muestran los resultados obtenidos en la investigación "Enseñanza de las didácticas en Colombia”, un punto de referencia conceptual y metodológico, y concretamente en el área de filosofía a partir de un trabajo desarrollado con cinco universidades del país. El trabajo indagó las perspectivas, que se sugieren a través de los documentos estudiados, sobre las nociones existentes con respecto al concepto de didáctica. La idea de trabajar con este concepto nace de la confusión que aún persiste en el ámbito educativo de asociar la didáctica a un enfoque instrumental o técnico, desconociendo los avances registrados a lo largo de los últimos años en los que se han constituido comunidades académicas que han discutido su estatus epistemológico como área disciplinar encargada de pensar las dinámicas específicas de la enseñanza y aprendizaje en múltiples disciplinas del conocimiento, siendo una de estas el área de la filosofía.

En ese sentido, para este primer apartado se pretende ubicar, inicialmente, las múltiples definiciones que se han brindado en torno a la didáctica por algunos autores representativos en el tema. Posteriormente, se habla de algunos avances registrados por comunidades académicas que han desarrollado investigaciones que dan cuenta de la constitución de esta disciplina emergente (la didáctica de las ciencias). Por último, se aborda la discusión desde el ámbito de la filosofía, advirtiendo que han existido trabajos que evidencian un desarrollo conceptual en torno a esta didáctica específica.

El término didáctica proviene del griego didaktiké, didaskein, didaskalia, didaktikos, didasko $(\delta \iota \delta \alpha \kappa \tau \iota \kappa \varepsilon, \delta \iota \delta \alpha \sigma \kappa \varepsilon \iota v, \delta \iota \delta \alpha \sigma \kappa \alpha \lambda \iota \alpha, \delta \iota \delta \alpha \kappa \tau \iota \kappa o \sigma$, $\delta \iota \delta \alpha \sigma \kappa o$ ) (Navarra, 2001) y alude a los verbos enseñar, instruir y exponer. En otras palabras, la didáctica desde sus raíces etimológicas se encuentra direccionada a pensar el quehacer práctico de la docencia, considerando el buen modo de abordar el conocimiento para su enseñanza. A partir de esta noción básica, se ha ido construyendo a lo largo de la historia su definición epistemológica. Esta definición, según De Camilloni (1998), tiene en sus inicios una base normativa. Así, Comenio (citado por De Camilloni et al., 1998) fue quien impulsó originalmente una reflexión sistemática sobre este concepto, concibiendo a la didáctica como una técnica de enseñanza universal; esto es, una técnica que permite enseñar todo a todos. En la misma línea, Herbart (citado por De Camilloni et al., 1998) sugiere que la didáctica es un proceso formal de instrucción que permite la transmisión del conocimiento de forma adecuada. Por su parte, De Camilloni et al. (2007) proponen pensarla como un medio que permite describir, explicar y establecer 
normas que dinamicen las acciones llevadas a cabo en la enseñanza.

Ya desde otras perspectivas, la didáctica se distancia de los modelos normativos e instrumentales para configurarse como ciencia o disciplina en el ámbito educativo. Esta perspectiva la comparte Navarra (2001), quien ve en la didáctica una "ciencia de la educación que estudia e interviene en el proceso de enseñanza/aprendizaje con el fin de conseguir la formación intelectual del educando" (p. 5). De la misma forma, Vasco (2008) la concibe como una disciplina con pretensiones de cientificidad, que reflexiona de forma sistemática los problemas que se sitúan en los interrogantes de cómo aprenden y qué se enseña a los alumnos. Para Zambrano (2005) la didáctica se asume como una disciplina científica cuyo estudio remite a las condiciones de enseñanza y aprendizaje; como a la génesis, circulación y apropiación del saber. Por su parte, Tamayo (2014) la asume como una disciplina con objeto de estudio, siendo este último los procesos de enseñanza/ aprendizaje; tiene además como objetivo central el desarrollo del pensamiento crítico en dominios específicos del conocimiento. Cabe destacar en este mismo autor, su propuesta de configurar la constitución de la didáctica a partir del aporte de seis esferas del conocimiento que albergan sus propios desarrollos teóricos y metodológicos, como lo son: la metacognición, las emociones, la evolución concetual, las representaciones, las relaciones ciencia, tecnología y sociedad y la historia epistemológica de las ciencias.

Expuesto lo anterior, además de una gama de significados sobre el concepto de didáctica, también es claro el desarrollo conceptual que se ha alcanzado en este campo: de tener una mirada con pretensiones de orden instrumentalista, a ser considerada una disciplina encargada de pensar los procesos de aprendizaje y enseñanza en componentes específicos del conocimiento. En ese sentido, las pretensiones de la didáctica se descentralizaron de la enseñanza y pasaron a contemplar otro tipo de componentes valiosos en el proceso educativo, como lo son el aprendizaje, los contenidos, el contexto y el pensa- miento crítico. Este logro alcanzado se dio en parte por la constitución de comunidades académicas que optaron por un acercamiento más minucioso del fenómeno específico del componente didáctico.

Uno de estos primeros acercamientos proviene del ya conocido concepto de transposición didáctica planteado por Chevallard (1991), quien establece la transformación de un saber sabio, producido en las esferas altas de la investigación científica, a un saber escolar, siendo este menos complejo pero guardando las implicaciones de rigurosidad que lo distinguen. Se trata, así, de moldear un conocimiento que permita ser comprendido a la luz de sus inicios epistemológicos.

Este desarrollo teórico contribuyó a que se pensara en los procesos de enseñanza en áreas específicas del conocimiento. A propósito, Shulman (1987) formula dicho concepto y como soporte afirma que cada área del conocimiento contiene unas dinámicas que le son propias, situación que revela la necesidad de didácticas que piensen estas particularidades en lo que él llamó conocimiento pedagógico de contenido, asumiéndose como la combinación efectiva entre el conocimiento disciplinar (o ciencia de referencia y objeto de enseñanza) y el conocimiento pedagógico.

De manera análoga, Bolívar (2005) concibe la importancia de contar con didácticas específicas que permitan reflexionar sobre las variables que inciden en los procesos de enseñanza, como: la edad, particularidades de los alumnos y los contenidos específicos del conocimiento. Igualmente, Montero y Vez (citados por Bolívar, 2005), sugieren que "la especificidad de los contenidos transforma y moldea los contenidos comunes" (p. 10). Adicional a estas ideas, Tamayo (2014) ve en este tipo de didácticas "una fuente inagotable de experiencias específicas que parten del reconocimiento de la naturaleza de la ciencia a enseñar y de las especificidades contextuales en las que se realiza el proceso de formación" ( $p$. 30). Y apartarnos de seguir buscando recetas o fórmulas mágicas para solucionar los problemas en el aula de clases (Ruiz, 2007). Bajo estas construcciones epistemológicas, surgieron las diferentes didácticas 
específicas, lo que dio origen a nuevos problemas de enseñanza, aprendizaje y pensamiento crítico en estos dominios particulares.

Una muestra de lo anterior ha sido la didáctica de las ciencias. Adúriz-Bravo en su trabajo $\mathrm{La}$ didáctica de las ciencias como disciplina (2000), plantea cómo esta didáctica ha tenido un desarrollo histórico y conceptual, logrando, de manera gradual, su consolidación como disciplina autónoma. Parte de esta evolución ha sido ubicada en etapas caracterizadas por algunos hechos que dieron cabida a su propio orden secuencial. Así, se habla de las etapas adisciplinar, tecnológica, protodisciplinar, emergente y consolidada. De esta forma, se propone que "la didáctica de las ciencias está marcada por un movimiento hacia grados crecientes de integración de las perspectivas teóricas fuentes (epistemológica, psicológica y pedagógica), de las que surge una perspectiva didáctica independiente, cada vez menos deudora de las anteriores" (p. 67).

Para Tamayo y Orrego (2003), la didáctica de las ciencias ha cumplido paulatinamente las condiciones requeridas para la consecución de una autonomía como disciplina, considerando que esta posee por sí misma un marco problematizador y un objeto propio de estudio que se encarga de describir, analizar y comprender los problemas más significativos de la enseñanza y el aprendizaje de las ciencias.

Por su parte, Astolfi (2001) concibe que la didáctica de las ciencias es una disciplina con un entramado conceptual ajeno a cualquier otro saber, puesto que sus fundamentos epistemológicos se delimitan a partir de la problemática específica que le rodea.

Constituida la didáctica de las ciencias como disciplina, esta ha identificado algunos problemas específicos en medio de sus procesos de enseñanza y aprendizaje, como: la argumentación en clase de ciencias y los procesos de autorregulación en su aprendizaje (Tamayo, 2014).

La didáctica, en conclusión, ha registrado un cambio en sus perspectivas. La concepción que inicialmente se registraba de ella como método y estrategia de enseñanza, se modificó hacia una perspectiva más amplia en la que se albergan elementos como el aprendizaje, los contenidos, el contexto y el pensamiento crítico. De allí la importancia que han tenido los trabajos de autores como Chevallard (1991), Shulman (1987), Bolívar (2005), Ruiz (2006) y Tamayo (2014); que abordan la didáctica desde la transposición, la especificidad y sus finalidades, dando una mayor apertura a su conceptualización. De igual forma, cabe resaltar la consolidación que ha tenido la didáctica en un campo disciplinar como la ciencia, pues esta se ha constituido en un área disciplinar autónoma que propicia la identificación de problemas educativos específicos en su propia esfera.

\section{Didáctica de la filosofía}

Ahora bien, las reflexiones en torno a la enseñanza de la filosofía se registran desde la antigüedad con Platón, pues las mismas meditaciones hechas en esta época reflejan preguntas como: “ ¿Se puede realmente incluir la didáctica a la filosofía como un instrumento externo, independiente del tema que ha de ser tratado? ¿No pertenece a la filosofía, ya desde Platón, preguntarse por su capacidad de mediación?" (Martens, 1983, p. 18).

En la modernidad, la pregunta por la enseñanza de la filosofía la hicieron ilustres pensadores como Kant y Hegel. En Pineda (2012) se encuentra una interpretación al respecto, pues dicho autor señala que Hegel tenía una profunda preocupación por la forma como se estaba brindando la enseñanza de la filosofía en los centros educativos, ya que esta se encontraba desprovista de contenidos propiamente filosóficos. Según el mismo autor, este hecho vino a darse por la mala interpretación otorgada a la máxima propuesta por Kant en la que se plantea que no se puede aprender filosofía, sino que solo se puede aprender a filosofar. Para Kant enseñar a filosofar no implicaba de ninguna forma el dejar de lado los contenidos relacionados a la filosofía.

Desde entonces, la producción en materia de reflexión sobre la enseñanza de la filosofía ha sido vasta. Así, en una etapa más contemporánea, los 
trabajos desarrollados en torno a este tema buscaron generar algunas reflexiones con respecto a las características que debía tener una didáctica de la filosofía. De igual forma, suscitaban una serie de propuestas metodológicas que permitieran el manejo pertinente en las formas de enseñanza filosófica. Al respecto, Bondy, en su obra Didáctica de la filosofía (1967), nos ilustra sobre las implicaciones que reviste pensar una didáctica especial en este ámbito del saber, proponiendo asimismo, algunos métodos que permitirían adecuar los procesos de enseñanza, según las perspectivas filosóficas asumidas. Para Mercant (1986), la didáctica de la filosofía ha confluido en tres tendencias que han posibilitado algunos métodos para su enseñanza, por ese motivo habló del método de filosofía para niños, método dialógico-pragmático y método interdisciplinar. El propósito de cada uno de estos métodos es el de buscar el mejoramiento de las prácticas de enseñanza de la filosofía bajo un espíritu crítico. En Perelló (2008), existe adicionalmente, una evidente preocupación por dotar a la enseñanza de la filosofía de estrategias que permitan generar una comprensión de sus dinámicas epistemológicas.

Ya en trabajos más recientes de autores como Obiols (2008) y Cerletti (2008), se propone que la didáctica no esté solamente delimitada a la consideración de unos de métodos específicos que permitan eludir los apuros de las prácticas educativas; esta debe también encargarse de pensar el conjunto de elementos que hacen posible su enseñanza. En la misma línea de pensamiento, algunos autores consideran que la didáctica de la filosofía debe procurar cuestionamientos que permitan configurar significados respecto a su enseñanza, de tal manera que al encontrar sentidos a estos interrogantes se puedan adecuar estrategias metodológicas acordes a los mismos (Boavida, 2006; Kohan, 2011).

En Colombia, también ha existido una serie de autores que han estudiado el desarrollo conceptual de esta didáctica. Por ejemplo, Gómez, en su obra Introducción a la didáctica de la filosofía (2003), concibe la importancia de darle un estatus epistemológico a esta disciplina, partiendo de las problemáticas específicas que aquejan al ámbito filosófico. En Araya (2003), la didáctica de la filosofía se constituye en el manejo de procedimientos metodológicos e instrumentales. El Ministerio de Educación Nacional en Colombia (MEN, 2010) ha hecho también algunos aportes otorgados a esta línea conceptual, presentando algunas definiciones al respecto, junto con algunas propuestas para su enseñanza; siendo esto ya un indicio de una perspectiva más tradicional. Por último, encontramos propuestas innovadoras como las de Oviedo y Restrepo (2013) en las que se busca relacionar las prácticas de enseñanza y aprendizaje de la filosofía con estudios hechos desde las neurociencias.

Visto esto, existe un marco amplio de reflexiones registradas en torno a la didáctica de la filosofía. Algunas con tendencias anudadas a perspectivas más tradicionalistas, por cuanto delimitan las reflexiones de la didáctica a lo metodológico; otras, por su parte, brindan una importancia especial al conjunto de las dinámicas específicas que deben considerarse para la enseñanza y el aprendizaje de la filosofía, dado que esta disciplina no es ajena a su contexto. En ese sentido, a pesar de las diferencias que puedan posicionarse en la forma como se adopte esta didáctica, ya existe una preocupación latente de una comunidad académica por pensar y discutir los problemas que subyacen a la práctica educativa desde el ámbito filosófico. Lo anterior permitiría debatir con mayor vehemencia aquellas posturas académicas que pretenden deslegitimar la discusión sobre la existencia de una didáctica específica de la filosofía, en tanto que aquellos que asumen estas posiciones lo hacen desde la consideración de que la enseñanza de la filosofía se debe ver como un problema más que aborda la filosofía.

En este orden de ideas, la didáctica de la filosofía es una necesidad imperiosa, máxime cuando debe ir ligada a los procesos de formación docente en esta misma área. Así, Gómez (2003) nos hacer ver que una formación en didáctica le permitiría al docente tener mayor claridad en las formas de enseñanza de conceptos filosóficos que en apariencia pueden concebirse como complejos para quienes apenas 
tienen un contacto incipiente con este saber. En la misma línea, Osorio (2006) concibe que la didáctica de la filosofía debe servirle al maestro para que este pueda efectuar la transición entre el saber erudito filosófico a un saber con los mismos matices, pero menos complejo. Ambas posiciones cercanas a la perspectiva de Chevallard comparten la necesidad de que la didáctica sea un campo de estudio que permita reducir las distancias entre aquellos planteamientos propiamente filosóficos y las posibilidades de aprendizaje de los alumnos.

Para Ruggiero (2012), la necesidad de una didáctica de la filosofía se sitúa en la posibilidad de que esta pueda abrir un espacio de reflexión, en el futuro docente, en torno al significado que reviste para este los conceptos de enseñanza y la propia filosofía. Todo esto con la intención de que las futuras prácticas educativas no carezcan de un sentido propiamente filosófico. Gómez (2006) y Cerletti (2008) asumen un planteamiento similar, pues estipulan que la construcción de significados en torno a la pregunta “¿qué es filosofía?”, pueden generar mayor claridad con respecto al manejo de las prácticas de enseñanza, y esto solo se lograría si hubiese una reflexión que parta de un eje articulador como la didáctica de la filosofía.

Ahora bien, desde nuestra perspectiva, la justificación por una didáctica de la filosofía ya viene determinada por el conjunto de elementos que estudia, de modo que se hace indispensable conocerlos en medio de un proceso de formación docente. Estos elementos se guían de una reflexión constante sobre las dinámicas concretas del conocimiento filosófico, los procesos de enseñanza/aprendizaje, los aspectos emocionales de los sujetos que enseñan y aprenden y de los aspectos relacionados con la autorregulación del aprendizaje. Todo esto con el fin de adecuar estrategias, según la perspectiva filosófica que se adopte, para llevar el conocimiento de la filosofía al individuo y fortalecer el desarrollo de procesos cognitivos superiores formativos como el pensamiento crítico, la argumentación, la autorregulación, la metacognición o el desarrollo de problemas.
Así las cosas, la propuesta de investigación que se ha desarrollado en este trabajo busca identificar, en los documentos escritos, las perspectivas teóricas en torno a la didáctica de la filosofía, considerando los planteamientos expuestos. Todo esto con el fin de poder situar dichas perspectivas y juzgar su pertinencia dentro de los programas de formación docente en filosofía.

\section{Metodología}

La investigación se llevó a cabo con la participación de cinco centros universitarios colombianos que dentro de su oferta educativa tienen programas de Licenciatura en Filosofía y Letras o Licenciatura en Filosofía que a 2018 tenían acreditación de alta calidad. En los programas con estas características, cinco universidades accedieron a participar y brindaron información necesaria para desarrollar esta investigación: Universidad del Valle, Universidad Tecnológica de Pereira, Universidad de Antioquia, Universidad de Caldas y Universidad de Nariño.

Para efectos de no ser reiterativos con los nombres de estas instituciones, se acude a una denominación de estas, a partir de las siguientes codificaciones: $\mathrm{U} 1$, Universidad del Valle; U2, Universidad Tecnológica de Pereira; U3, Universidad de Antioquia; U4, Universidad de Caldas; U5, Universidad de Nariño.

Cada institución brindó, previa solicitud, los programas de estudio pertenecientes a la asignatura o asignaturas en didáctica de la filosofía. Posterior a ello, se analizaron algunos elementos que componen la estructura de dichos programas, como justificación, objetivos, metodologías de clase, criterios de evaluación y referencias bibliográficas. Para el caso del presente artículo, solo se presentan los análisis $y$ reflexiones que surgieron en torno a los componentes asociados a justificación y objetivos.

Ahora bien, el trabajo investigativo tiene un enfoque cualitativo bajo un corte descriptivo comprensivo. Lo anterior en vista de que este tipo de investigación permite el análisis del discurso escrito de documentos cuyas estructuras albergan una serie de intenciones constituidas por quienes han 
sido artífices de su construcción. De esta manera, la técnica de estudio implementada fue el análisis documental, cuyo manejo permite "ahondar en las motivaciones e inclinaciones de los autores de las informaciones, sus formas de percibir la realidad, sus inclinaciones afectivas, ideológicas, cognitivas y con ellas se desprende información implícita que también afecta al contenido informativo de cualquier documento" (Peña y Pirela, 2007, p. 57). O como lo señala Lopez Vega (citado por García, 1993) brinda un "conjunto de operaciones que permiten desentrañar del documento la información en él contenida" (p. 12). Para complementar el análisis se aplicó el procedimiento de codificación abierta propuesto por Strauss y Corbin (2002) trabajado desde el software para análisis cualitativo de datos denominado Atlas.ti 8, con el cual se elaboraron las redes semánticas que contribuyeron a las asociaciones de las categorías centrales de estudio con los diferentes registros de los programas.

Así, el análisis documental de los programas se abordó bajo la dinámica de tres instancias distintas que permitieron generar claridad en la constitución de las categorías de investigación, como se muestra en la figura 1.

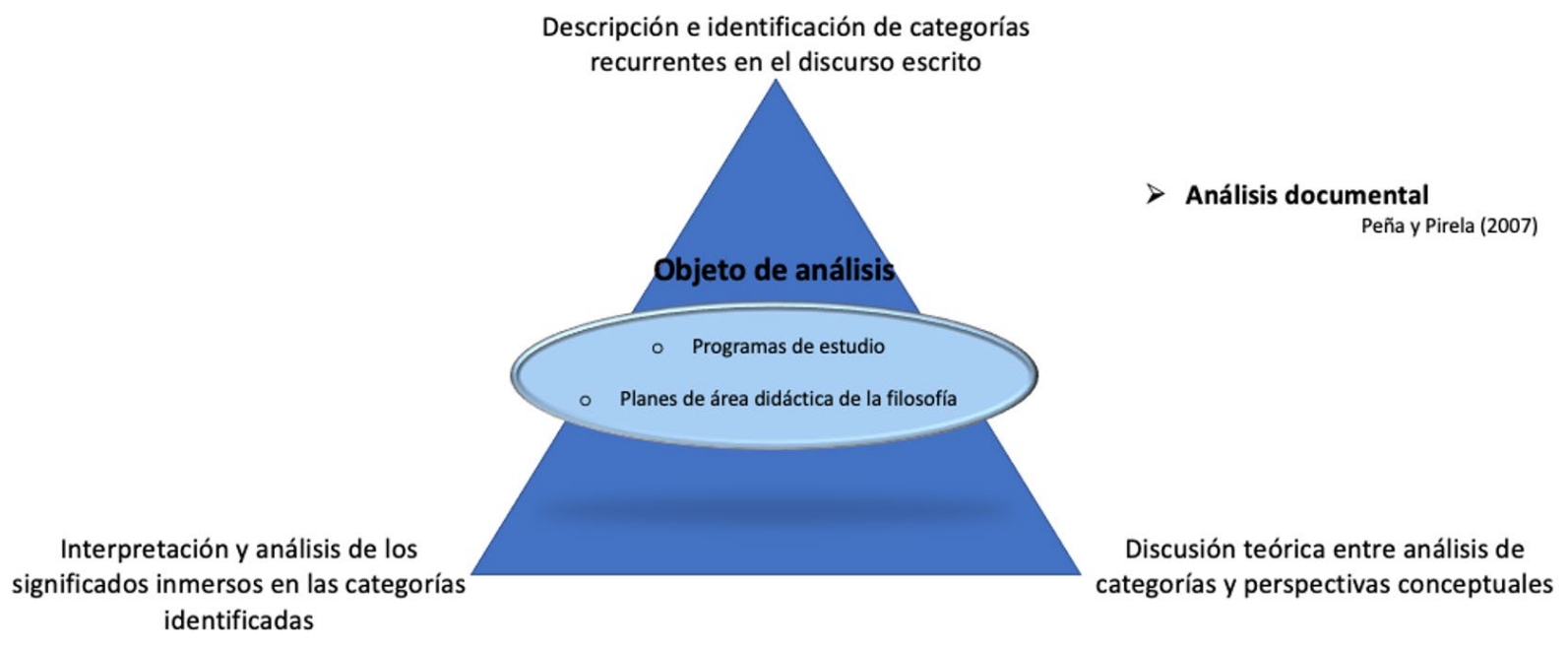

Figura 1. Proceso de análisis en documentos de programas académicos en didáctica de la filosofía

Fuente: elaboración propia.

En primer lugar, se efectuó una descripción de los registros identificados de los programas, fundamentando las dos categorías centrales de la investigación: perspectiva clásica de la didáctica y perspectiva contemporánea de la didáctica. En segundo lugar, se hizo un análisis interpretativo en torno a las ideas inmersas en dichos registros, con el fin de relacionar sus intenciones y significados a las categorías centrales de la propuesta. Por último, se abordó la discusión entre los datos analizados, categorías y componente teórico, buscando esta- blecer horizontes interpretativos que permitieran brindar mayor precisión y fundamentación a las conclusiones que arrojaba el estudio.

\section{Resultados}

Los resultados que se registran en este análisis se sitúan sobre el eje de las dos categorías mencionadas anteriormente y las relaciones obtenidas después de las maneras de análisis adoptadas. 


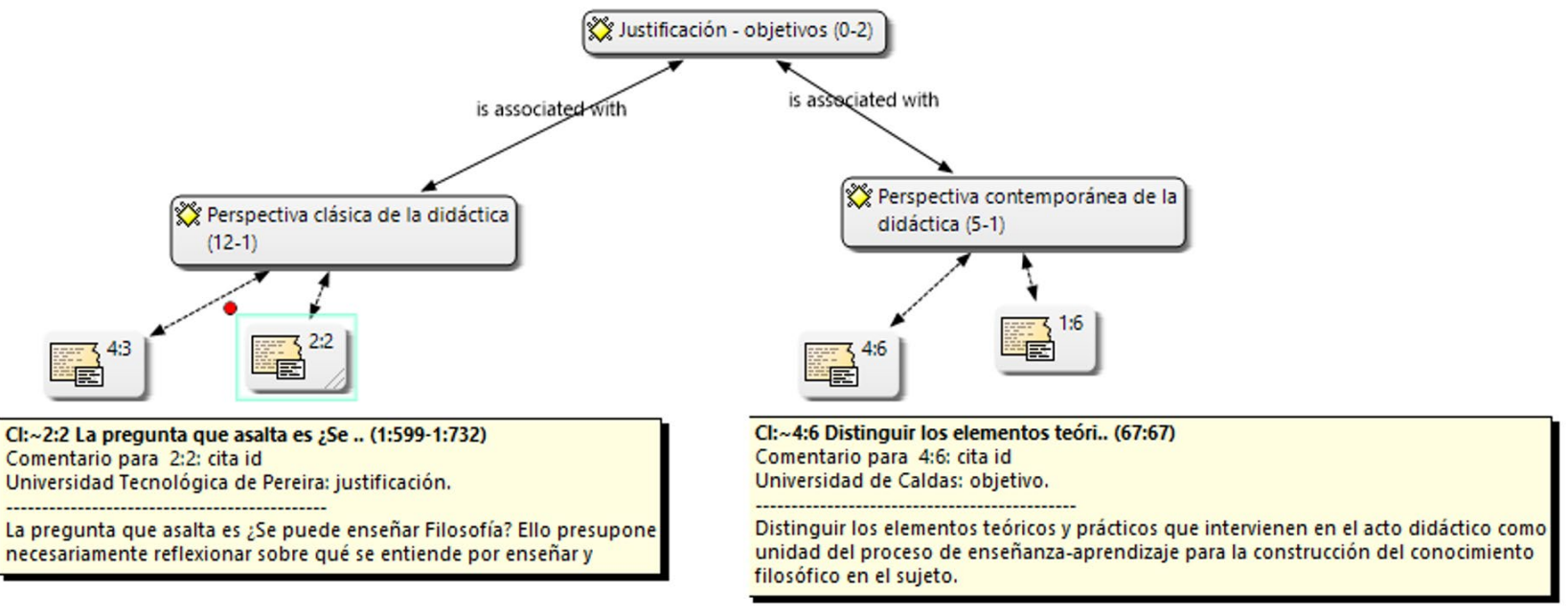

Figura 2. Presentación de las categorías de análisis derivadas de las secciones de los programas de estudio

Fuente: elaboración propia con apoyo del software Atlas.ti.

En la figura 1 se evidencian las correlaciones entre los dos ejes relevantes dentro de los planes de estudio desde la perspectiva de las secciones denominadas justificación y objetivos. De allí se derivan las categorías centrales de estudio: perspectiva clásica en didáctica y perspectiva contemporánea en didáctica. En ambas se encuentran unidades de registro que solo corresponden a las secciones de los programas, ya antes mencionadas.

En la primera de las conexiones de la red semántica que hace referencia a la categoría perspectiva clásica de la didáctica, se identificaron doce registros, como se ilustra en la figura 2.

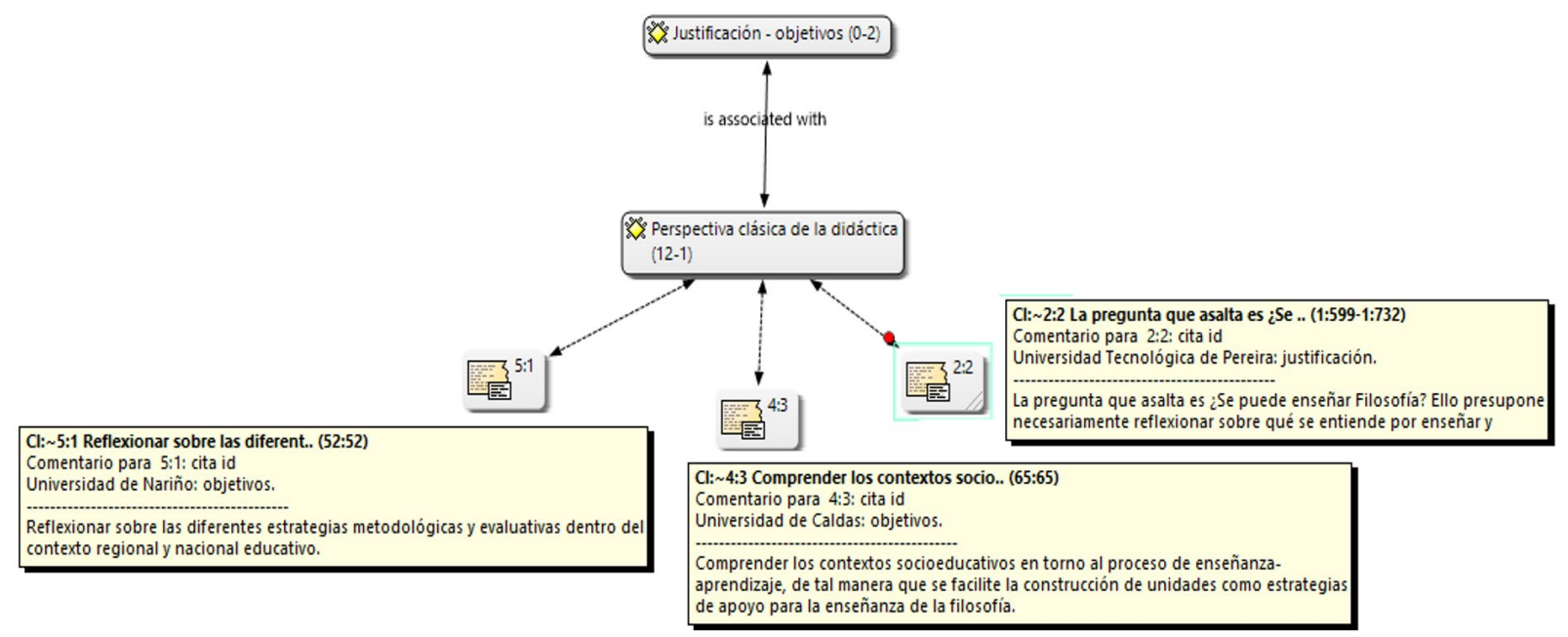

Figura 3. Citaciones relacionadas con conceptos expuestos por la perspectiva clásica de la didáctica

Fuente: elaboración propia con apoyo del software Atlas.ti. 
Los elementos asociados a esta red y que corresponden a la perspectiva clásica de la didáctica contienen ocho registros y ellos, a su vez, aparecen dentro de los currículos y programas de cuatro de las universidades: U2, U3, U4 y U5. La U2, por ejemplo, contempla como objetivo: "Dotar al estudiante de elementos conceptuales y herramientas didácticas que le permitan formular metodologías de clase afines a la naturaleza de la Filosofía". De igual forma lo establece la U3 cuando expresa: "Promover la discusión sobre los métodos y prácticas utilizadas en la enseñanza de la filosofía". Para el caso de la U4 se habla de "aplicar estrategias y técnicas de evaluación que favorezcan el aprendizaje significativo de la filosofía". Por último, en la U5 se busca "reflexionar sobre las diferentes estrategias metodológicas y evaluativas dentro del contexto regional y nacional educativo".

Como se observa, la intencionalidad semántica de los registros refleja el uso de estrategias, metodologías, técnicas y prácticas; tendencia que refleja un modelo tecnificador de la didáctica en el que se busca acoplar las prácticas educativas a un concierto de propuestas metodológicas. Esta visión no está muy lejos de lo que expresa Cerletti (2008), cuando alude a las necesidades académicas imperantes de los futuros docentes en cualquier área del conocimiento, pues "hay una demanda usual hacia la formación docente -por cierto común a casi todas las disciplinas- que podría graficarse, de manera simplificada, en algunos tópicos: necesito herramientas para dar clase, quiero instrumentos para poder enseñar" (p. 3).

Esta perspectiva ajustada a una visión normativa de la didáctica (Comenio, 1998), deja ver las limitaciones conceptuales que muchos docentes en ejercicio poseen en torno a este mismo concepto. Lo anterior se sustenta en prácticas investigativas como la hecha por Miranda y Badillo (2006), quienes indagaron a un grupo de docentes de la Universidad Pedagógica Nacional, por los fundamentos de conceptos como pedagogía y didáctica. En una de sus preguntas se quiso saber si los maestros pensaban que la didáctica no se reduce a un conjunto de algoritmos. Gran parte de las respuestas indicaron lo contrario de la afirmación; es decir, algunos maestros sí consideraban la didáctica como un saber meramente instrumental.

En ese sentido, este primer acercamiento sugiere una interpretación que somete algunas intenciones discursivas de los programas académicos en didáctica de la filosofía, a la construcción de prácticas centradas en la aplicación de métodos y estrategias prediseñadas, visión clásica de la didáctica.

Ahora bien, un segundo aspecto abordado en los registros identificados bajo esta misma categoría desarrollada corresponde a la noción de enseñanza. La didáctica ha ampliado su definición conceptual y, por ende, su campo de estudio, situando aspectos de orden cognitivo como el aprendizaje y la formación del pensamiento crítico (Navarra, 2001; Zambrano, 2005; Vasco, 2008; Tamayo, 2014). De esta forma, no sería posible establecer prácticas propicias de enseñanza, si antes no hay un conocimiento previo en torno a las formas de aprendizaje de los alumnos, dando lugar a un engranaje completo del proceso didáctico (Tamayo, Zona y Loaiza, 2015).

En los programas de estudio, muy contrario a lo planteado, se identifican siete registros en donde se alude de manera unilateral a la noción de enseñanza. Tres son las universidades en donde se halló algún tipo de evidencia al respecto: U1, U2, U3. Así, dando lugar a una muestra de lo anterior, la U1 en su justificación expresa: "Se piensa en todo caso, como un punto de partida para un abordaje, en clave filosófica, del saber y las prácticas pedagógicas y de la problematización de la enseñanza de la filosofía". Bajo las mismas condiciones se expone, como uno de sus objetivos, "abordar la relación entre enseñanza y filosofía”. En la U2, se encuentra que uno de los planteamientos en su justificación gira en torno a "La pregunta que asalta es $i S e$ puede enseñar Filosofía? Ello presupone necesariamente reflexionar sobre qué se entiende por enseñar...”. Ya en uno de sus objetivos se plantea "formular microclases donde el estudiante realice propuestas didácticas para la enseñanza de la Filosofía”. Por último, la U3 entre sus objetivos establece: "Orientar 
los procesos de formación de los estudiantes de la licenciatura en filosofía, con miras a reflexionar sobre las propuestas contemporáneas en didáctica y metódicas que pueden ser aplicadas a la enseñanza de la filosofía en nuestro medio".

En cada una de las unidades de registros analizadas, se identifican intencionalidades que se constituyen a partir del concepto de enseñanza; ya sea desde la problematización con la filosofía o a partir de la implementación de estrategias metodológicas. Sin embargo, no hay un intento por abordar la noción de aprendizaje de manera consciente y explícita. En este sentido, dichos objetivos sugieren una visión instrumental; pues, como lo plantea Kohan (2011), la problematización en la didáctica de la filosofía implicaría asociar conceptos como enseñanza y aprendizaje, esto para evitar posturas y prácticas que se centren en lo instrumental.

Esta manera de situar la didáctica, desde el discurso escrito, atendería también a la visión con respecto a la forma en que se relaciona este concepto con la pedagogía. Tradicionalmente se ha asociado a la didáctica como un saber derivado de la pedagogía que responde a la pregunta por el cómo (Tamayo, 2014). De allí que en muchas oportunidades se asocie un poco más en los discursos educativos el concepto de enseñanza, que la misma noción de aprendizaje. Esta interpretación no sugiere directamente que en los programas pueda haber un desconocimiento de la didáctica como ciencia, pero pueden existir confusiones o posturas que la sitúan dentro de los ámbitos de estudio de la pedagogía.

Un buen ejemplo que refuerza esta interpretación lo da el siguiente registro identificado en la justificación del programa de la U3. Este dice:

El enfoque del presente curso busca armonizar las propuestas de la formación filosófica en la educación inicial con las aspiraciones sociales y culturales en torno a las humanidades y su papel formativo, además, busca construir una didáctica de la filosofía a partir del diálogo que ésta sostiene con la pedagogía y no en las concepciones psicológicas sobre la infancia y la adolescencia exclusivamente. (Universidad de Antioquia, 2017, p 1)
Aquí se presenta un elemento coyuntural que desborda los fundamentos epistemológicos de la didáctica. Se habla de armonizar las propuestas filosóficas de formación junto con las aspiraciones sociales y culturales que se tienen desde las humanidades. Este planteamiento busca configurar las finalidades formativas que respondan a la pregunta: ¿Qué tipo de ser humano deseamos para la sociedad? Para un teórico como Zambrano (2016) estos interrogantes implican pensar los propósitos propuestos para la educación, lo cual supone, a su vez, abordar un sector importante en el campo de estudio de la pedagogía, pues para este, "la pedagogía es la reflexión del acto de educar" (p. 9). En Tamayo (2014) las reflexiones que se llevan a cabo en los procesos formativos en educación se fundamentan en la esfera de la pedagogía, dado que su objeto de estudio es precisamente la formación humana.

En este sentido, en este registro se puede identificar un matiz muy cercano al ámbito pedagógico, perspectiva que daría lugar a un distanciamiento en torno a propuestas contemporáneas desde el enfoque didáctico.

A manera de síntesis del análisis desarrollado bajo la categoría: perspectiva clásica en didáctica. Se puede establecer que hay un número de registros significativos que fundamentan una manera de ver la didáctica con un sentido clásico. Lo anterior dado que en el discurso escrito se reiteran conceptos como estrategias, metodologías, herramientas y enseñanza, y que son propios de una concepción instrumentalista que arraiga las prácticas educativas desde un sentido unilateral, excluyendo procesos cognitivos que se abren desde la esfera del aprendizaje. De igual forma, se aprecia que en algunos registros existen ciertos elementos asociados al componente pedagógico, pues se asumen reflexiones encaminadas a buscar los fines que se pretenden alcanzar con la educación desde la sociedad y la filosofía.

Ahora bien, en una segunda categoría de análisis de los programas se identificaron algunos elementos que son propios de una didáctica contemporánea, como: el aprendizaje, el pensamiento crítico y la 
Universidad Pedagógica Nacional

Facultad de Humanidades

distinción entre teoría y práctica. Así, se halla cinco registros en donde se puede presentar alguna de estas caracterizaciones.

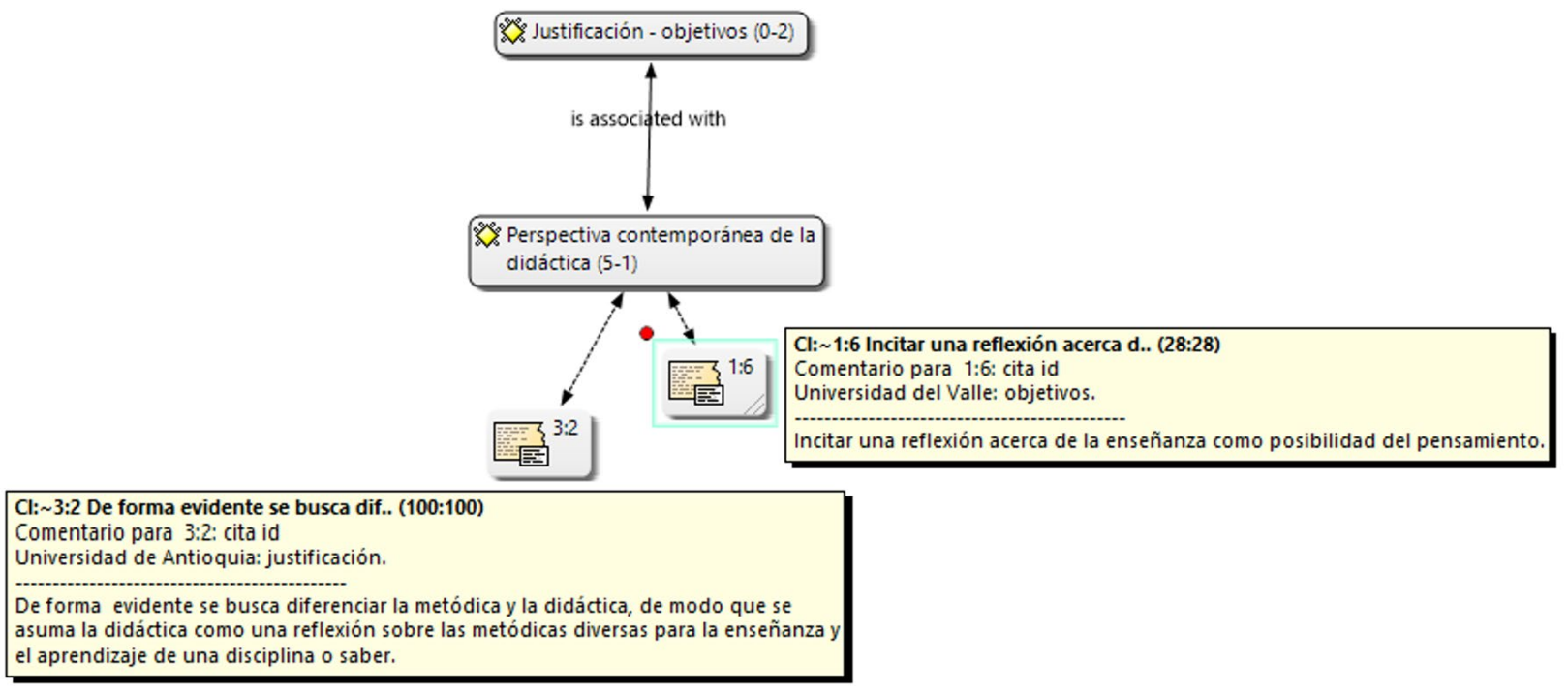

Figura 4. Citaciones relacionadas con conceptos expuestos por la perspectiva contemporánea de la didáctica

Fuente: elaboración propia con apoyo del software Atlas.ti.

Un primer elemento que se destaca dentro de las unidades de registro es la posibilidad de incitar al pensamiento crítico. El desarrollo de este elemento es imprescindible en la escuela, de allí que cada área del conocimiento debe procurar su potencialización en quienes están en medio del proceso educativo. En el caso de la filosofía, por su condición inherente al análisis y la reflexión, tiene como deber exigir una formación direccionada al razonamiento crítico de los hechos, deber que implica vincular en los programas de didáctica intencionalidades que estén acordes al desarrollo del pensamiento. Así, en estos mismos, solo se identificó un registro bajo estas connotaciones en la U1, este versa: "Incitar una reflexión acerca de la enseñanza como posibilidad del pensamiento". La configuración semántica del registro busca establecer una actividad reflexiva que permita, a través de la enseñanza, impulsar prácticas transformadoras del pensar.

Como se evidencia, hay una carencia en las intencionalidades discursivas de los programas en didáctica por atender de manera explícita uno de los objetivos de la formación filosófica: el pensar. Para Boavida (2006), los conceptos filosóficos no tienen en sí mismos un poder problematizador que pueda llevar al límite el pensamiento, de allí que se requiera y se justifique una didáctica específica de la filosofía que permita recrear dichas problemáticas en razón de esta finalidad. Por su parte, teóricos como Bondy (1967), Gómez (2003) y Cerletti (2008) entienden que las prácticas educativas asociadas a la enseñanza y el aprendizaje de la filosofía deben romper los límites impuestos por las barreras sociales y culturales, teniendo como punto de referencia la práctica del filosofar. Entendiendo esta última como el cuestionamiento constante que lleva a asumir una forma de pensar. Así las cosas, este tipo de propuestas están en consonancia con lo que Tamayo, Zona y Loaiza (2015) proponen en el marco de la enseñanza y el aprendizaje de cualquier disciplina; y con lo que Tamayo (2014) plantea como objeto de estudio de las didácticas específicas: formar en pensamiento crítico. 
En consecuencia, los programas de estudio en didáctica específica de la filosofía deben procurar emprender acciones direccionadas a la construcción de prácticas educativas que promuevan el desarrollo del pensamiento crítico, pues no es suficiente con el manejo conceptual de una historia filosófica. Se deben problematizar los conceptos y hacer que estos puedan llegar al límite de la pregunta. De esta forma, una didáctica específica de la filosofía puede legitimarse en sus condiciones y en su propio objeto de estudio.

Ahora bien, una segunda línea de análisis que se propone bajo esta categoría viene determinada por la distinción que se hace entre teoría y práctica en la didáctica. Si bien es cierto que este planteamiento no se esboza dentro de las líneas generales del marco teórico como un elemento perteneciente a la visión contemporánea de la didáctica, su señalamiento es interesante en tanto que podría vislumbrar una superación de las concepciones y prácticas educativas que ven en la didáctica un elemento metodológico.

Tres son las universidades que junto con sus registros albergan alguna de estas caracterizaciones: a) la U2 propone en uno de sus objetivos: "Dotar al estudiante de elementos conceptuales y herramientas didácticas que le permitan formular metodologías de clase afines a la naturaleza de la Filosofía y al contexto al cual se dirige el proceso de enseñanza/ aprendizaje"; b) la U3 pretende "de forma evidente [...] diferenciar la metódica y la didáctica, de modo que se asuma la didáctica como una reflexión sobre las metódicas diversas para la enseñanza y el aprendizaje de una disciplina o saber"; y c) la U4 establece: "Distinguir los elementos teóricos y prácticos que intervienen en el acto didáctico como unidad del proceso de enseñanza/aprendizaje para la construcción del conocimiento filosófico en el sujeto". Cada registro, como se constata, sugiere una intención por delimitar el campo teórico y práctico de la didáctica; ya sea en la construcción de unidades didácticas o en la elección de estrategias metodológicas apropiadas para el proceso educativo.
Desde los planteamientos conceptuales ya se han establecido los fundamentos sobre los que se debe constituir el objeto de estudio de la didáctica. Elementos como la enseñanza, el aprendizaje, el pensamiento crítico y la metacognición son de necesaria reflexión para su implementación en la práctica escolar. De allí que, si estos elementos son analizados desde un componente teórico, podría ampliarse la concepción de una didáctica que ha estado amparada en un interrogante: el cómo.

Cabe destacar que en los programas se debería precisar los supuestos sobre los que se fundamenta la teoría de la didáctica, de tal manera que hubiese aún mayor claridad en torno a qué variables se tienen por importantes cuando se pretenden construir unidades didácticas o estrategias metodológicas.

Un último aspecto por reseñar en la categoría que se desarrolla se relaciona con uno de los análisis hechos en la categorización anterior: los conceptos de enseñanza y aprendizaje. A pesar de que existe un número importante de registros en donde se asocia la didáctica a la noción de enseñanza, también lo es que hay intenciones discursivas, en menor proporción, en donde se configura la dualidad del proceso vinculando el aprendizaje. Cuatro son los registros que albergan estás intencionalidades, ya tres de ellos son los que se han presentado anteriormente. Un cuarto registro lo presenta la U4 cuando indica: "Aplicar estrategias y técnicas de evaluación que favorezcan el aprendizaje significativo de la filosofía".

Dentro del discurso escrito de los programas de estudio debería existir una intención por relacionar de forma constante las nociones de enseñanza y aprendizaje. Así, se configuraría el acto educativo en el que se comprende las dinámicas divergentes en los aprendizajes de los alumnos, previo a la conformación de las formas en que se podrían hacer las intervenciones sobre la práctica de la enseñanza. Este movimiento singular, acogido bajo el nombre de didáctica, proporcionaría los fundamentos necesarios para afianzar el desarrollo de capacidades como el pensamiento crítico. 
Así pues, la identificación de algunos registros bajo ciertos matices contemporáneos de la didáctica permitiría pensar que en los discursos escritos de los programas se están brindando cambios en algunas concepciones sobre este concepto, a pesar de que predominan ciertas nociones clásicas que hacen ver de esta un medio para la consecución de un fin. De esta forma, elementos como el aprendizaje y el pensamiento crítico deben fortalecerse dentro del hilo discursivo y la práctica, además de otras nociones igualmente importantes como metacognición, emociones y representaciones (Tamayo, 2014); para que se pueda puntualizar los problemas y fortalezas que se presentan en la especificidad de la enseñanza/ aprendizaje de la filosofía.

\section{Conclusiones}

En la presentación de los análisis que se hicieron sobre los programas de estudio se resaltan algunas tendencias en torno a una visión clásica de la didáctica. Conceptos como estrategias, metodologías y enseñanza predominan en el marco de las intenciones discursivas de estos programas, hecho que llevaría a interpretar la existencia de posibles formas de concebir el proceso educativo de manera unilateral; es decir, bajo un enfoque en el que predomina las prácticas activas del docente sin contar las particularidades que se dinamizan en los aprendizajes de los alumnos. En ese sentido, fueron pocos los registros que se identificaron con la intención de establecer el concepto de aprendizaje como aspecto relevante en medio del proceso educativo.

Por su parte, también se hallaron intenciones discursivas acordes a los elementos que configuran el objeto de estudio de la pedagogía, pues se atiende a reflexiones encaminadas a encontrar los fines que tendría la educación en la escuela y desde el área de filosofía.

Asimismo, se resalta la existencia de solo algunos elementos encontrados que proveen al discurso de los programas una caracterización más contemporánea de la didáctica. Ideas como la distinción entre teoría y práctica en el componente didáctico y el pensamiento crítico, podrían contribuir a una ampliación de las perspectivas epistemológicas que se tienen sobre esta disciplina.

Así las cosas, en los programas de estudio de la cátedra en didáctica de la filosofía impera la idea de abordar estrategias metodológicas que permitan el conocimiento y la práctica de la enseñanza; de hecho, no se reconocen con amplitud elementos que son también importantes para el proceso educativo y que se posicionan desde el mismo discurso contemporáneo de la didáctica. Este panorama sugiere que desde la didáctica de la filosofía deban profundizarse aspectos como el aprendizaje, la metacognición y el mismo pensamiento crítico, con el fin de consolidar la formación de futuros maestros y constituir una estructura epistemológica apropiada a una didáctica específica de la filosofía.

En este sentido, se requieren estudios que permitan evaluar el desarrollo epistémico de esta didáctica específica a partir de los componentes contemporáneos que la caracterizan. También, se deben abordar investigaciones en las cuales se pueda identificar potenciales relaciones entre los planteamientos escritos y los aconteceres en el aula de clase, esto permitiría conocer la coherencia entre las estructuras teóricas y el desempeño de las prácticas en clase en la asignatura didáctica de la filosofía.

\section{Referencias}

Aduriz-Bravo, A. (2000). La didáctica de las ciencias como disciplina. Enseñanza, 17(18), 61-74.

Araya, D. (2003). Didáctica de la filosofía. Cooperativa Editorial Magisterio.

Astolfi, J. (2001). Conceptos clave en la didáctica de las disciplinas: referencias, definiciones y bibliografías de didáctica de las ciencias. Díada.

Boavida, J. (2006). De una didáctica de la filosofía a una filosofía de la educación. Revista Española de Pedagogía, 234, 205-226.

Bolívar, A. (2005). Conocimiento didáctico del contenido y didácticas específicas. Profesorado. Revista de Currículum y Formación del Profesorado, 9(2), 1-39.

Bondy, A. (1967). Didáctica de la filosofía. Arisca.

Cerletti, A. (2008). La enseñanza de la filosofía como problema filosófico. Libros del Zorzal. 
Chevallard, Y. (1991). La transposición didáctica. Del saber sabio al saber enseñado. Ed. Aique.

Comenio, J. (1998). Didáctica Magna. Editorial Porrúa.

De Camilloni, A., Cols, E., Basabe, L. y Feeney, S. (2007). El saber didáctico. Paidós.

De Camilloni, A., Davini, M., Edelstein, G., Litwin, E., Souto, M. y Barco, S. (1998). Corrientes didácticas contemporáneas. Paidós.

García, A. C. (1993). Análisis documental: el análisis formal. Revista General de Información y Documentación, 3(1), 11-19.

Gómez, A. (2006). ¿… Enseñar filosofía? Universidad del Valle.

Gómez, M. (2003). Introducción a la didáctica de la filosofía. Pereira: Editorial Papiro.

Kohan, W. (2011). Desafíos para pensar... la enseñanza de la filosofía. Cuestiones de Filosofía, 11. https://doi. org/10.19053/01235095.v0.n11.2009.649

Martens, E. (1983). Introducció a la didàctica de la filosofía. Valencia: Universitat de València.

Mercant, S. (1986). Tendencias actuales de la didáctica de la Filosofía. Taula: Quaderns de Pensament, 6, 141-146.

Ministerio de Educación Nacional (MEN). (2010). Orientaciones pedagógicas para la filosofía en la educación media. Bogotá. https://www.mineducacion.gov.co/

Miranda, R. y Badillo, R. (2006). Concepciones sobre pedagogía y didáctica de un grupo de docentes. Informe de investigación. Revista Educación y Pedagogía, 18(44), 127-137.

Navarra, J. (2001). Didáctica: concepto, objeto y finalidades. En F. Sepúlveda y N. Rajadell (coords.), Didáctica general para psicopedagogos (pp. 25-60). Universidad Nacional de Educación a Distancia - UNED

Obiols, G. (2008). Una introducción a la enseñanza de la filosofía. Libros del Zorzal.

Osorio, A. (2006). Filosofía, filosofía de la educación y didáctica de la filosofía. Universidad de Caldas.

Oviedo, D. y Restrepo, V. (2013). Enseñanza de la filosofía en Colombia: hacia un enfoque multisensorial en el campo didáctico. Nodos y Nudos, 4(34), 37-48. https://doi.org/10.17227/01224328.2282.

Peña, T. y Pirela, J. (2007). La complejidad del análisis documental. Información, Cultura y Sociedad, 16, 55-81.
Perelló, J. (2008). Didáctica de la Filosofía. Revista Sophia, Colección de Filosofía de la Educación, 4, 155-210.

Pineda, D. (2012). Hegel: Sobre la enseñanza de la filosofía. Universitas Philosophica, 29(59). 139-159.

Ruggiero, G. (2012). La formación de los profesores de filosofía u la paradoja del "giro filosófico". Educar em Revista, 46, 99-112.

Ruiz, F. (2006). Ideas de ciencia y su incidencia en el proceso de enseñanza-aprendizaje. Revista Latinoamericana de Estudios Educativos, 2(1), 119-130.

Ruiz, F. (2007). Modelos didácticos para la enseñanza de las ciencias naturales. Revista Latinoamericana de Estudios Educativos, 3(2), 41-60.

Shulman, L. (1987). Conocimiento y enseñanza: fundamentos de la nueva reforma. Profesorado. Revista de Currículum y Formación del Profesorado, 9(2), 1-30.

Strauss, A. y Corbin, J. (2002). Bases de la investigación cualitativa: técnicas y procedimientos para desarrollar la teoría fundamentada. Editorial Universidad de Antioquia.

Tamayo, O. (2014). Pensamiento crítico dominio-específico en la didáctica de las ciencias. Tecné, Episteme y Didaxis: TED, 36, 25-45. https://doi. org/10.17227/01203916.4686

Tamayo, O. y Orrego, M. (2003). Caracterización general de la didáctica. Doctorado en Ciencias de la Educación de Rudecolombia. Cade, Universidad de Caldas.

Tamayo, O., Zona, R. y Loaiza, Y. (2015). El pensamiento crítico en la educación. Algunas categorías centrales en su estudio. Revista Latinoamericana de Estudios Educativos, 11(2), 111-133.

Universidad de Antioquia. (2017). Programa del curso Didáctica II Filosofía.

Vasco, C. (2008). Educación, pedagogía y didáctica. En G. H. Vásquez (ed.), Filosofía de la educación (pp. 99-127). Editorial Trotta.

Zambrano, A. (2005). Didáctica, pedagogía y saber. Cooperativa Editorial Magisterio.

Zambrano, A. (2016). Pedagogía y didáctica: esbozo de las diferencias, relaciones, tensiones de dos campos. Praxis \& Saber, 7(13), 45-61. 